\title{
Does External Debt Service Devalue Local Currency in the Long Run? Empirical Evidence from Egypt
}

\author{
Emad Omar Elhendawy ${ }^{1}$ \\ ${ }^{1}$ University of Jeddah, College of Business at Khulis, Department of Finance, Jeddah, Saudi Arabia \\ Correspondence: Emad Omar Elhendawy, Associate Professor, University of Jeddah, College of Business at \\ Khulis, Department of Finance, Jeddah, Saudi Arabia. Tel: 966-56-300-5205.
}

Received: November 30, 2021

Accepted: January 6, 2022

Online Published: January 7, 2022

doi:10.5539/ijef.v14n2p51

URL: https://doi.org/10.5539/ijef.v14n2p51

\begin{abstract}
The aim of this study is to identify the extent to which there is an effect of external debt service on the exchange rate in Egypt in the long run, where the change in the exchange rate has great importance in changing currency value and thus affecting its function as a store of value and a standard for forward payments and then in the redistribution of income and wealth, It also has an effect on some macroeconomic variables, such as inflation, exports, imports, and thus the current account. The study examines the estimation of the long-run relationship between the external debt service and the exchange rate in Egypt in the period 1980-2019 and relies on the exchange rate of the dollar against the Egyptian pound as a dependent variable, while the explanatory variables were the external debt service, gross capital formation, broad money growth, deposit interest rate, household final consumption expenditure, gross savings, and terms of trade adjustment. The methodology is based on Vector Error Correction (VEC) and the study concluded that there is a significant long-term relationship between the value of the Egyptian pound and all the variables explained in the study, as the error correction coefficient is negative and significant. Also, there is an inverse statistically significant relationship between the value of the Egyptian pound and each of the external debt service, the deposit interest rate, and gross savings; any change of $1 \%$ in the external debt service, the deposit interest rate, and gross savings leads to a devaluation of the Egyptian pound against the dollar by $4.8 \%, 0.04 \%$, and $0.05 \%$, respectively. The study also concluded that there is a positive, statistically significant relationship in the long term between the value of the Egyptian pound and each of gross capital formation, broad money growth, households' and NPISHs' final consumption expenditure, and terms of trade adjustment, as any change of $1 \%$ in these variables leads to an increase in the value of the Egyptian pound by $0.16 \%, 0.05 \%, 0.27 \%$, and $6 \%$, respectively. This study recommends that decision makers consider all the reasons that would reduce the external debt service in order to preserve the value of the Egyptian currency in the long run.
\end{abstract}

Keywords: Egypt, External Debt Service, exchange rate, devaluation, Vector Error Correction

\section{Introduction}

There is extensive literature on the benefits of public debt in foreign currencies. The most important potential benefits of foreign currency debt include access to a larger investor base, reduced crowding out of private sector lending in domestic markets or repeat inflationary financing (Bua, Juan, \& Andrea, 2014), lower returns on foreign currency issuance, access to longer maturities, ability to build official foreign currency reserves and improved short-term stability term longevity in difficult times. Also, in developing countries, the state resorts to external borrowing to provide the private sector's needs of foreign exchange, whether by lending where companies cannot access foreign money markets or by guaranteeing loans. Moreover, external borrowing in foreign currencies may lead to more discipline fiscal and monetary, whereby the government's incentive to create inflation decreases to reduce the value of the local currency. But foreign currency financing is risky and tends to be volatile, cyclical and subject to sudden stops (Cavallo \& Tavella, 2013). The issuance of foreign currency on a large scale can increase the external vulnerability of a country as seen by investors and credit rating agencies. The presence of foreign currency debt, along with real exchange rate volatility increases the volatility of GDP growth and capital inflows (Eichengreen, Hausmann, \& Panizza, 2005a).

Also, a significant depreciation of the local currency may significantly increase the interest burden as calculated in that currency. In addition, external debt may cause problems in the economy; it increases the country's 
exposure to external conditions, especially when debt is contracted at a variable rate and with rising global interest rates, which leads to an increase in debt service costs. A depreciation also leads to increased debt service (in terms of the domestic currency), and when the country borrows to cover the growing deficit, external borrowing leads to an unsustainable level of debt, an excessive share of debt service in total government spending, and a significant use of foreign currency to service debt which may lead to a debt crisis in the long term (Beaugrand, Loko, \& Mlachila, 2002). As for external borrowing for the purpose of formation of foreign exchange reserves. If foreign money is sterilized, it leads to the same effects of domestic loans as increasing interest rates and excluding private investment. If money is not sterilized, external financing is accompanied by increased domestic demand and thus results in pressure on inflation or the balance of payments (Beaugrand, Loko, \& Mlachila, 2002). The rest of this study is organized as follows: Section 2 gives the Empirical Review. Section 3 presents the methodology and model estimation. Finally section 4 gives the results and conclusion.

\section{Empirical Review}

Most of the empirical studies agreed on the existence of an inverse significant relationship between the value of the currency and the service of the external public debt, despite covering different periods of time and different explanatory variables and their application to different countries. The following table 1 shows a summary of the results of some of these studies.

Table 1. Summary of some empirical studies

\begin{tabular}{ll}
\hline Study & Methodology \\
\hline Ajayi, R. A.,\& Jongmoo, J.C. (1993). & This paper proposes a structural \\
model that is an aggregation of an addition to the usual variables such as money \\
asset and a monetary model of supply and interest rates, has a largely \\
exchange rates along the Frankel Line significant and negative impact on the external \\
(1983), modified to include external values of most of the countries' currencies.
\end{tabular}

Aderemi, Timothy Ayomiunde et al. (2020). study checked The relationship between external debt and exchange rate fluctuations in Nigeria during the period from 1981 to 2018.

Alam, Noor and Fauziah Md. Taib (2013). this study designs a model wherein the relationship of external public debt with budget deficit, current account deficit, and exchange rate depreciation are empirically tested. The study is dichotomy that covers empirical analysis of panels of a group of six "Debt Trap Countries (DTC)" namely as, India, Indonesia, Nepal, Pakistan, Sri Lanka, and Thailand and eight "Non Debt Trap Countries (NDTC)" as Bangladesh, Fiji, Korea, Malaysia, Myanmar, Papua New Guinea, Philippines, and Singapore, of Asian Pacific Developing Countries (APDC).The study period of thirty years (1971 to 2000).

Mutua et al. (2020). The research examined the impact of external debt service on the exchange rate in Kenya. Times series data was used for the period 1982 to 2016. debt. Estimate this model for a sample of 18 LDCs.

This study used an Autoregressive Distribution Lag model (ARDL), and the variables used exchange rate fluctuations as dependent variables, and external debt, debt service payment, and foreign reserves as independent variables.

In model, the benefit of autoregressive (dynamic) modeling has been taken as granted for using the option of lagged value(s) of the regress and as explanatory variable.

The Vector Autoregression model was estimated using first difference of the variables. The Impulse Response Functions and variance decomposition were estimated.
External debt as a means of financing the budget deficit which has been discouraged in Nigeria in the short term because its servicing and repayment especially puts pressure on the foreign exchange market in the short term and thus leads to exchange rate fluctuations in terms of depreciation of nairan in Nigeria.

The results showed a positive relationship between the external public debt, the budget deficit, the current account deficit, and the exchange rate depreciation.

The external debt service had a negative impact on the exchange rate in the short-run in Kenya. 
Titus Freeman Ifeanyi, Nwanne \& Richard, Eze. (2015). The aim of this study is to investigate the relationship between external public debt servicing and receipt and exchange rate fluctuations in Nigeria from 1981 to 2013.

Kouladoum Jean-Claude ; Dombou T. Dany R. (2019). The objective of this work is to analyze the effect of external debt on the real exchange rate in Chad from 1975 to 2014.

Odera, Q. A. (2015). This study empirically investigated the effects of external public debt on real effective exchange rate (REER) volatility in Kenya under the complete float regime for period 1993 to 2013.
The variables were used in this study included external public debt receipts, external public debt servicing, and exchange rate Fluctuations. This study used multiple regressions (Ordinary Least Square) and Co-integration to establish the short-run and long-run relationship.

The generalized method of moment is used in this study

A linear model was used and exchange rate volatility was regressed against inflation, interest rates, and GDP growth rate, money supply to GDP ratio and external debt to GDP ratio using the Ordinary Least Square technique.
The results of the study showed that foreign debt receipts and foreign debt service have short and long-term positive relationships with exchange rate fluctuations in Niran. The study concluded that while the receipts of the external public debt positively affect the exchange rate, the service of the external public debt negatively affects the exchange rate.

The results showed that debt service affects negatively and significantly real exchange rate in Chad.

The results showed that external debt to GDP ratio had negative and significant effect on REER volatility in Kenya.

\section{Methodology and Model Estimation}

This study examines the estimation of the relationship between Exchange rate and Debt service on external debt in Egypt in the period 1980-2019 in long run. The study relied as a dependent variable on the Exchange rate of the dollar against the Egyptian pound (the number of dollar units of the pound), while the explanatory variables were the Debt service on external debt, Gross capital formation, Broad money growth, Deposits interest rate, Household final consumption expenditure, Gross Savings and Terms of trade adjustment. The methodology is based on both the Vector Error Correction (VEC) through Eviews 12.

$$
\begin{gathered}
\text { EXCHANGE }=\alpha+\beta 1 \text { DEBTSERVICE }+\beta 2 \text { CAPITAL }+\beta 3 \text { M2GROWTH }+\beta 4 \text { INTEREST }+ \\
\beta 5 \text { HOUSEHOLD }+\beta 6 \text { GSAVINGS }+\beta 7 \text { TOT }+\varepsilon
\end{gathered}
$$

Where:

EXCHANGE: Exchange Rates, US dollar per domestic currency.

M2: Broad money growth (annual \%).

DEBTSERVICE: Debt service on external debt, public and publicly guaranteed (PPG) (TDS, current US\$)

CAPITAL: Gross capital formation (\% of GDP).

M2GROWTH: Broad money growth (annual \%).

INTEREST: Deposit interest rate (\%).

HOUSEHOLD: Households and NPISHs final consumption expenditure (\% of GDP).

GSAVINGS: Gross savings (\% of GDP).

TOT: Terms of trade adjustment (constant LCU).

$\varepsilon$ : White-noise error term.

The data obtained from the World Bank website (https://data.worldbank.org/indicator/DT.TDS.DPPG.CD) and IMF (International Financial Statistics).

Table 2 shows the descriptive statistics for the study variables. It is clear that the data for all variables follow a normal distribution by the Jarque-Bera test where the null hypothesis was accepted except for the exchange rate and debt service variables. The data are distributed as a normal distribution where there is a probability $>$ 0.05 for all study variables except for the exchange rate and debt service variables where the probability is $<$ 0.05 . 
Table 2. Descriptive statistics for the variables of the study

\begin{tabular}{lcccccccc}
\hline & EXCHANGE & DEBTSERVICE & CAPITAL & M2GROWTH & INTEREST & HOUSEHOLD & GSAVINGS & TOT \\
\hline Mean & 0.500 & $2.66 \mathrm{E}+0$ & 21.48 & 83.4867 & 9.46651 & 74.0503 & 20.82133 & $5.45 \mathrm{E}+10$ \\
Median & 0.280 & $2.08 \mathrm{E}+0$ & 19.52 & 82.5967 & 9.64687 & 73.9320 & 20.68581 & $5.12 \mathrm{E}+10$ \\
Maximum & 1.430 & $7.85 \mathrm{E}+0$ & 33.11 & 98.1361 & 12.3166 & 88.1239 & 35.47563 & $1.46 \mathrm{E}+11$ \\
Minimum & 0.060 & $6.23 \mathrm{E}+0$ & 13.64 & 66.4234 & 6.01666 & 63.0986 & 9.593465 & $-1.07 \mathrm{E}+10$ \\
Std. Dev. & 0.526 & $1.68 \mathrm{E}+0$ & 5.753 & 7.73518 & 2.05608 & 6.05974 & 6.433382 & $3.53 \mathrm{E}+10$ \\
Skewness & 1.128 & 1.73614 & 0.671 & 0.26238 & -0.26739 & 0.21691 & 0.343591 & 0.679766 \\
Kurtosis & 2.440 & 5.26205 & 2.125 & 2.66614 & 1.64205 & 2.71667 & 2.804237 & 3.385836 \\
Jarque-Bera & 9.003 & 28.6227 & 4.281 & 0.64474 & 3.55001 & 0.44748 & 0.850905 & 3.328663 \\
Probability & 0.011 & 0.00000 & 0.117 & 0.72442 & 0.16948 & 0.79952 & 0.653474 & 0.189317 \\
Sum & 20.020 & $1.07 \mathrm{E}+1$ & 859.5 & 3339.46 & 378.660 & 2962.01 & 832.8533 & $2.18 \mathrm{E}+12$ \\
Sum Sq. Dev. & 10.788 & $1.10 \mathrm{E}+2$ & 1291.01 & 2333.48 & 164.871 & 1432.10 & 1614.148 & $4.85 \mathrm{E}+22$ \\
Observations & 40 & 40 & 40 & 40 & 40 & 40 & 40 & 40 \\
\hline
\end{tabular}
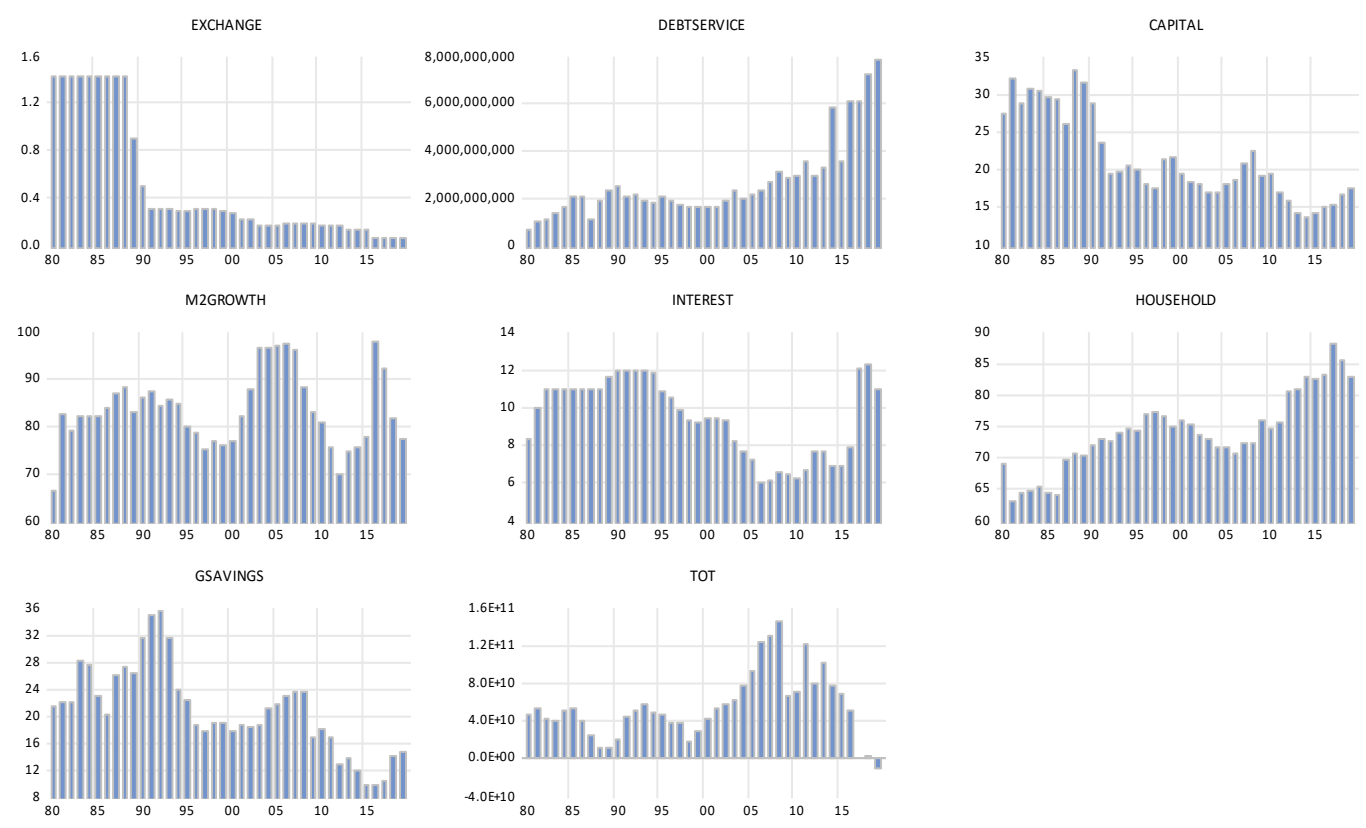

Figure 1. The graph of the study variables

\subsection{Unit Root Test}

The following Table 3 shows the results of the time series stability test will use the breakpoint unit root test will beuse. It is clear that all the variables are stable in the first difference with a constant enabling us to carry out Co-integration of study variables.

Table 3. Summary of results of the Unit root test

\begin{tabular}{|c|c|c|c|c|c|}
\hline \multirow{3}{*}{ variables } & \multicolumn{3}{|c|}{ Test Augmented Dickey Fuller (ADF) } & & \\
\hline & \multicolumn{2}{|c|}{ Level } & \multicolumn{3}{|c|}{ First Difference } \\
\hline & Constant & Trend & Constant & Trend & Summery \\
\hline EXCHANGE & $-2.35^{* *}$ & -1.91 & $-2.23 * *$ & -2.56 & $\mathrm{I}(1)$ \\
\hline DEBTSERVICE & 3.35 & 3.26 & -0.565 & $-10.37 *$ & $\mathrm{I}(1)$ \\
\hline CAPITAL & -1.50 & -2.23 & $-6.57^{*}$ & $-6.66^{*}$ & $\mathrm{I}(1)$ \\
\hline M2GROWTH & 0.04 & -2.57 & $-6.14 *$ & $-6.03^{*}$ & $\mathrm{I}(1)$ \\
\hline INTEREST & 0.16 & -1.93 & $-4.99 *$ & $-4.89 *$ & $\mathrm{I}(1)$ \\
\hline HOUSEHOLD & 0.96 & -0.77 & $-7.07 *$ & $-7.48^{*}$ & $\mathrm{I}(1)$ \\
\hline GSAVINGS & -0.71 & -1.25 & $-4.95^{*}$ & $-4.90 *$ & $\mathrm{I}(1)$ \\
\hline TOT & -1.18 & -1.61 & $-6.77 *$ & $-6.71 *$ & $\mathrm{I}(1)$ \\
\hline
\end{tabular}

Note. 1) For ADF and PP tests, ***, ** and * denote rejection of a unit root hypothesis based on Mackinnon (1991) critical values at $10 \%, 5 \%$ and1percentage respectively. 


\subsection{Co-Integration Test}

From Table 4 which shows the results of Co- integration test, it is clear that there is three models for the Cointegration of study variables that enables us to use the Vector error correction model to estimate the long-term relationship between Exchange rate, Debt service on external debt, Gross capital formation, Broad money growth, Deposits interest rate, Household final consumption expenditure, Gross Savings and Terms of trade adjustment.

Table 4. The result of Cointegration test

\begin{tabular}{ccccc}
\hline HypothesizedNo. of CE(s) & Eigenvalue & Trace Statistic & 0.05 Critical Value & Prob.** \\
\hline None * & 0.88 & 261.04 & 159.53 & 0.00 \\
At most $1 *$ & 0.83 & 180.04 & 125.62 & 0.00 \\
At most 2* & 0.71 & 113.06 & 95.75 & 0.00 \\
At most 3 & 0.52 & 66.01 & 69.82 & 0.10 \\
At most 4 & 0.45 & 38.50 & 47.86 & 0.28 \\
At most 5 & 0.16 & 16.02 & 29.80 & 0.71 \\
At most 6 & 0.13 & 9.48 & 15.49 & 0.32 \\
At most 7 & 0.11 & 4.37 & 3.84 & 0.04 \\
\hline
\end{tabular}

Trace test indicates 3 cointegrating eqn(s) at the 0.05 level $*$ denotes rejection of the hypothesis at the 0.05 level

**MacKinnon-Haug-Michelis (1999) p-values.

\begin{tabular}{|c|c|c|c|c|}
\hline HypothesizedNo. of CE(s) & Eigenvalue & Max-EigenStatistic & 0.05 Critical Value & Prob.** \\
\hline None * & 0.88 & 81.00 & 52.36 & 0.00 \\
\hline At most $1 *$ & 0.83 & 66.99 & 46.23 & 0.00 \\
\hline At most $2 *$ & 0.71 & 47.04 & 40.08 & 0.01 \\
\hline At most 3 & 0.52 & 27.51 & 33.88 & 0.24 \\
\hline At most 4 & 0.45 & 22.47 & 27.58 & 0.20 \\
\hline At most 5 & 0.16 & 6.54 & 21.13 & 0.97 \\
\hline At most 6 & 0.13 & 5.11 & 14.26 & 0.73 \\
\hline At most $7 *$ & 0.11 & 4.37 & 3.84 & 0.04 \\
\hline
\end{tabular}

Max-eigenvalue test indicates 3 cointegrating eqn(s) at the 0.05 level $*$ denotes rejection of the hypothesis at the 0.05 level

**MacKinnon-Haug-Michelis (1999) p-values.

\subsection{Vector Error Correction (VEC)}

The results of the long-term relationship between Exchange rate, Debt service on external debt, Gross capital formation, Broad money growth, Deposits interest rate, Household final consumption expenditure, Gross Savings and Terms of trade adjustment were estimated in the equation (2) and in Table 5.

$$
\begin{aligned}
& \text { EXCHANGE }=-26.37-4.80 D E B T S E R V I C E+0.16 C A P I T A L+0.06 M 2 G R O W T H-0.04 I N T E R E S T \\
& +0.271 \text { HOUSEHOLD -0.054GSAVINGS + 6.07TOT }
\end{aligned}
$$

From the equation 2 and Table 5 bellow, it is clear to us through the estimated relationship in the model that: First: There is a significant long-term relationship between the value of the Egyptian pound against the dollar and all the variables explained in the study, as the error correction coefficient is negative and significant. Second: There is an inverse statistically significant relationship between the value of the Egyptian pound and each of the debt service on external debt, the deposit interest rate and Gross savings, as any change of $1 \%$ in the debt service on external debt, the deposit interest rate and Gross savings leads to a devalue of the Egyptian pound against the dollar by $4.8 \%, 0.04 \%$ and $0.05 \%$ respectively. Third: There is a positive statistically significant relationship in the long term between the value of the Egyptian pound against the dollar and each of Gross capital formation, Broad money growth, Households and NPISHs final consumption expenditure and Terms of trade adjustment, as any change of $1 \%$ in these variables leads to an increase in The value of the Egyptian pound against the dollar by $0.16 \%, 0.05 \%, 0.27 \%$ and $6 \%$, respectively.

The value of coefficient $\mathrm{R}^{2}$ reached $87.5 \%$, meaning that the independent variables in the model explain an amount of $87.5 \%$ of the change in the value of the Egyptian pound against the dollar. 
Table 5. VEC estimates

\begin{tabular}{|c|c|c|c|c|c|c|c|c|}
\hline \multicolumn{2}{|l|}{ Cointegrating Eq: } & \multicolumn{3}{|c|}{ CointEq1 } & & & & \\
\hline \multicolumn{2}{|l|}{ EXCHANGE(-1) } & \multicolumn{2}{|c|}{1.000000} & & & & & \\
\hline \multirow{3}{*}{\multicolumn{2}{|c|}{ DEBTSERVICE(-1) }} & & & & & & \\
\hline & & \multirow{2}{*}{\multicolumn{2}{|c|}{$\begin{array}{c}(1.8 \mathrm{E}-11) \\
{[-27.1889]}\end{array}$}} & & & & & \\
\hline & & & & & & & & \\
\hline \multirow{2}{*}{\multicolumn{2}{|c|}{ CAPITAL(-1) }} & \multicolumn{2}{|c|}{0.160618} & & & & & \\
\hline & & \multicolumn{2}{|c|}{$(0.01029)$} & & & & & \\
\hline & & \multicolumn{2}{|c|}{ [ 15.6156$]$} & & & & & \\
\hline \multicolumn{2}{|l|}{ M2GROWTH(-1) } & \multicolumn{2}{|c|}{0.057483} & & & & & \\
\hline & & \multicolumn{2}{|c|}{$(0.00225)$} & & & & & \\
\hline & & {$[25$} & 5339] & & & & & \\
\hline INTEREST(-1) & & -0.0 & 4933 & & & & & \\
\hline & & (0. & 1898) & & & & & \\
\hline & & {$[-2$} & 6679] & & & & & \\
\hline HOUSEHOLD(-1) & & 0.2 & 1421 & & & & & \\
\hline & & (0. & 1068) & & & & & \\
\hline & & {$[25$} & 4073] & & & & & \\
\hline GSAVINGS(-1) & & -0.0 & 4814 & & & & & \\
\hline & & $(0.1$ & $522)$ & & & & & \\
\hline & & {$[-10$} & 5007] & & & & & \\
\hline TOT(-1) & & & $\mathrm{E}-12$ & & & & & \\
\hline & & & E-12) & & & & & \\
\hline & & {$[4.7$} & 192] & & & & & \\
\hline $\mathrm{C}$ & & -26 & 5668 & & & & & \\
\hline & & $\mathrm{D}(\mathrm{EX}$ & HANGE) D(DE & BTSE... & & & & \\
\hline Error Correction: & D(EXCHANGE) & D(DEBTSE... & D(CAPITAL) & D(M2GROW... & D(INTEREST) & $\mathrm{D}($ HOUSEH... & D(GSAVINGS) & $\mathrm{D}$ (TOT) \\
\hline CointEq1 & -0.194925 & $-2.17 \mathrm{E}+08$ & -0.546743 & 7.404341 & -0.001972 & -1.603608 & 4.667484 & $-2.02 \mathrm{E}+09$ \\
\hline & $(0.04852)$ & $(4.4 \mathrm{E}+08)$ & $(1.75530)$ & $(3.56521)$ & $(0.72426)$ & $(1.76483)$ & $(2.10540)$ & $(2.2 \mathrm{E}+10)$ \\
\hline & {$[-4.01743]$} & {$[-0.49004]$} & {$[-0.31148]$} & [ 2.07683] & {$[-0.00272]$} & {$[-0.90864]$} & [ 2.21691] & {$[-0.09203]$} \\
\hline D(EXCHANGE(-1)) & 0.852944 & $1.34 \mathrm{E}+09$ & 2.406341 & -8.034553 & 0.028012 & -0.020262 & -10.14400 & $-1.05 \mathrm{E}+11$ \\
\hline & $(0.14074)$ & $(1.3 \mathrm{E}+09)$ & $(5.09142)$ & $(10.3413)$ & (2.10078) & (5.11908) & $(6.10692)$ & $(6.4 \mathrm{E}+10)$ \\
\hline & [ 6.06057] & [ 1.04423$]$ & {$[0.47263]$} & {$[-0.77694]$} & [ 0.01333$]$ & {$[-0.00396]$} & {$[-1.66107]$} & {$[-1.65607]$} \\
\hline D(EXCHANGE(-2)) & -0.173569 & $8.06 \mathrm{E}+08$ & 12.96385 & 17.68694 & 0.127030 & -0.183225 & 2.963982 & $2.46 \mathrm{E}+10$ \\
\hline & $(0.14915)$ & $(1.4 \mathrm{E}+09)$ & $(5.39569)$ & (10.9593) & $(2.22632)$ & $(5.42500)$ & $(6.47187)$ & $(6.7 \mathrm{E}+10)$ \\
\hline & {$[-1.16374]$} & [ 0.59165$]$ & [ 2.40263] & [ 1.61388$]$ & {$[0.05706]$} & {$[-0.03377]$} & [ 0.45798$]$ & [ 0.36468] \\
\hline D(DEBTSERVICE & $-2.15 \mathrm{E}-11$ & -0.818529 & $-5.98 \mathrm{E}-10$ & $-8.01 \mathrm{E}-10$ & $2.63 \mathrm{E}-10$ & $7.77 \mathrm{E}-11$ & $1.23 \mathrm{E}-10$ & -7.509688 \\
\hline$(-1))$ & $(2.0 \mathrm{E}-11)$ & $(0.18442)$ & $(7.3 \mathrm{E}-10)$ & $(1.5 \mathrm{E}-09)$ & $(3.0 \mathrm{E}-10)$ & $(7.3 \mathrm{E}-10)$ & $(8.8 \mathrm{E}-10)$ & (9.12878) \\
\hline & {$[-1.06618]$} & {$[-4.43836]$} & {$[-0.81917]$} & {$[-0.53997]$} & {$[0.87250]$} & {$[0.10581]$} & {$[0.14050]$} & {$[-0.82264]$} \\
\hline D(DEBTSERVICE & $2.66 \mathrm{E}-11$ & -0.057777 & $-2.42 \mathrm{E}-10$ & 3.69E-09 & $-1.45 \mathrm{E}-10$ & $-4.54 \mathrm{E}-10$ & $9.90 \mathrm{E}-11$ & -3.591259 \\
\hline$(-2))$ & $(1.9 \mathrm{E}-11)$ & $(0.17322)$ & $(6.9 \mathrm{E}-10)$ & $(1.4 \mathrm{E}-09)$ & $(2.8 \mathrm{E}-10)$ & $(6.9 \mathrm{E}-10)$ & $(8.2 \mathrm{E}-10)$ & $(8.57450)$ \\
\hline & [ 1.40229$]$ & {$[-0.33354]$} & {$[-0.35281]$} & [ 2.65084$]$ & {$[-0.51364]$} & {$[-0.65778]$} & [ 0.12031$]$ & {$[-0.41883]$} \\
\hline D(CAPITAL $(-1))$ & 0.002875 & 25105362 & -0.136364 & -1.645009 & -0.013471 & 0.134431 & -0.642058 & $1.05 \mathrm{E}+09$ \\
\hline & $(0.00892)$ & $(8.2 \mathrm{E}+07)$ & $(0.32283)$ & $(0.65570)$ & $(0.13320)$ & $(0.32458)$ & $(0.38722)$ & $(4.0 \mathrm{E}+09)$ \\
\hline & {$[0.32221]$} & [ 0.30789$]$ & {$[-0.42240]$} & {$[-2.50878]$} & {$[-0.10113]$} & [ 0.41417$]$ & {$[-1.65814]$} & [ 0.26072] \\
\hline D(CAPITAL $(-2))$ & 0.032337 & $1.58 \mathrm{E}+08$ & 0.001305 & -1.173394 & 0.038893 & 0.348479 & -0.410893 & $-5.38 \mathrm{E}+09$ \\
\hline & $(0.00851)$ & $(7.8 \mathrm{E}+07)$ & $(0.30796)$ & $(0.62550)$ & $(0.12707)$ & $(0.30963)$ & $(0.36938)$ & $(3.9 \mathrm{E}+09)$ \\
\hline & [ 3.79873] & [ 2.03355] & {$[0.00424]$} & {$[-1.87593]$} & [ 0.30608$]$ & [ 1.12546$]$ & {$[-1.11238]$} & {$[-1.39801]$} \\
\hline D(M2GROWTH(-1)) & 0.012823 & 85830775 & 0.047778 & 0.120499 & 0.070007 & 0.105999 & -0.173528 & $-1.44 \mathrm{E}+09$ \\
\hline & $(0.00335)$ & $(3.1 \mathrm{E}+07)$ & $(0.12115)$ & $(0.24606)$ & $(0.04999)$ & $(0.12180)$ & $(0.14531)$ & $(1.5 \mathrm{E}+09)$ \\
\hline & [ 3.82909] & [ 2.80497] & {$[0.39438]$} & {$[0.48971]$} & [ 1.40051$]$ & {$[0.87024]$} & {$[-1.19420]$} & {$[-0.95142]$} \\
\hline D(M2GROWTH(-2)) & -0.002644 & 5896693. & 0.158567 & -0.129844 & -0.039765 & -0.075782 & 0.086906 & $4.35 \mathrm{E}+08$ \\
\hline & $(0.00259)$ & $(2.4 \mathrm{E}+07)$ & $(0.09366)$ & $(0.19024)$ & $(0.03865)$ & $(0.09417)$ & $(0.11235)$ & $(1.2 \mathrm{E}+09)$ \\
\hline & {$[-1.02131]$} & [ 0.24925$]$ & [ 1.69294$]$ & {$[-0.68252]$} & {$[-1.02894]$} & {$[-0.80471]$} & {$[0.77356]$} & [ 0.37159] \\
\hline D(INTEREST(-1)) & 0.036182 & $2.85 \mathrm{E}+08$ & -0.827983 & -2.052522 & 0.608567 & 0.993765 & -0.540004 & $-6.04 E+09$ \\
\hline & $(0.01723)$ & $(1.6 \mathrm{E}+08)$ & $(0.62349)$ & $(1.26637)$ & $(0.25726)$ & $(0.62687)$ & $(0.74784)$ & $(7.8 \mathrm{E}+09)$ \\
\hline & [ 2.09940] & [ 1.80947] & {$[-1.32799]$} & {$[-1.62079]$} & [ 2.36559] & [ 1.58527$]$ & {$[-0.72208]$} & {$[-0.77502]$} \\
\hline D(INTEREST(-2)) & -0.005051 & $1.77 \mathrm{E}+08$ & 0.285577 & 1.453278 & -0.408548 & -0.631532 & 0.877648 & $-3.81 \mathrm{E}+09$ \\
\hline & $(0.01473)$ & $(1.3 \mathrm{E}+08)$ & $(0.53300)$ & $(1.08257)$ & $(0.21992)$ & $(0.53589)$ & $(0.63930)$ & $(6.7 \mathrm{E}+09)$ \\
\hline & {$[-0.34285]$} & [ 1.31470$]$ & {$[0.53580]$} & [ 1.34243$]$ & {$[-1.85771]$} & {$[-1.17847]$} & [ 1.37282$]$ & {$[-0.57236]$} \\
\hline כ(HOUSEHOLD(-1); & 0.019758 & 71068385 & 0.557260 & -2.386504 & -0.021339 & 0.290612 & -1.111277 & $-2.21 E+09$ \\
\hline & $(0.01193)$ & $(1.1 \mathrm{E}+08)$ & $(0.43175)$ & $(0.87693)$ & $(0.17814)$ & $(0.43409)$ & $(0.51786)$ & $(5.4 \mathrm{E}+09)$ \\
\hline & [ 1.65555$]$ & [ 0.65169$]$ & [ 1.29071$]$ & {$[-2.72143]$} & {$[-0.11978]$} & [ 0.66947$]$ & {$[-2.14590]$} & [-0.41007] \\
\hline
\end{tabular}




\begin{tabular}{|c|c|c|c|c|c|c|c|c|}
\hline \multirow[t]{3}{*}{ כ(HOUSEHOLD(-2), } & 0.017988 & $2.44 \mathrm{E}+08$ & 0.140056 & -0.719733 & -0.022429 & 0.174155 & -0.804611 & $-5.72 \mathrm{E}+09$ \\
\hline & $(0.00934)$ & $(8.5 \mathrm{E}+07)$ & $(0.33797)$ & $(0.68645)$ & $(0.13945)$ & $(0.33980)$ & $(0.40538)$ & $(4.2 \mathrm{E}+09)$ \\
\hline & [ 1.92546$]$ & [ 2.85493] & [ 0.41441$]$ & {$[-1.04848]$} & {$[-0.16084]$} & {$[0.51252]$} & {$[-1.98485]$} & {$[-1.35417]$} \\
\hline \multirow[t]{3}{*}{ D(GSAVINGS(-1)) } & 0.009650 & 10402508 & 0.270555 & -0.036814 & 0.013467 & 0.031884 & 0.263426 & $1.18 \mathrm{E}+09$ \\
\hline & $(0.00450)$ & $(4.1 \mathrm{E}+07)$ & $(0.16269)$ & $(0.33045)$ & $(0.06713)$ & $(0.16358)$ & $(0.19514)$ & $(2.0 \mathrm{E}+09)$ \\
\hline & [2.14583] & [0.25314] & [ 1.66296$]$ & {$[-0.11141]$} & [0.20062] & [ 0.19491] & [ 1.34990] & {$[0.57850]$} \\
\hline \multirow[t]{3}{*}{ D(GSAVINGS(-2)) } & -0.010606 & 9876284 & 0.003379 & -0.024277 & 0.023349 & -0.241107 & -0.271530 & $1.51 \mathrm{E}+09$ \\
\hline & $(0.00397)$ & $(3.6 \mathrm{E}+07)$ & $(0.14365)$ & $(0.29176)$ & $(0.05927)$ & $(0.14443)$ & $(0.17230)$ & $(1.8 \mathrm{E}+09)$ \\
\hline & {$[-2.67105]$} & {$[0.27221]$} & {$[0.02352]$} & {$[-0.08321]$} & [ 0.39395$]$ & {$[-1.66942]$} & {$[-1.57595]$} & [ 0.83811$]$ \\
\hline \multirow[t]{3}{*}{ D(TOT(-1)) } & $-4.84 \mathrm{E}-13$ & 0.006586 & $-5.39 \mathrm{E}-12$ & $-4.85 \mathrm{E}-12$ & $-4.76 \mathrm{E}-12$ & $2.90 \mathrm{E}-11$ & $-4.36 \mathrm{E}-11$ & -0.493005 \\
\hline & $(6.3 \mathrm{E}-13)$ & $(0.00580)$ & $(2.3 \mathrm{E}-11)$ & $(4.7 \mathrm{E}-11)$ & $(9.5 \mathrm{E}-12)$ & $(2.3 \mathrm{E}-11)$ & $(2.8 \mathrm{E}-11)$ & $(0.28716)$ \\
\hline & {$[-0.76267]$} & [ 1.13530$]$ & {$[-0.23480]$} & {$[-0.10406]$} & {$[-0.50272]$} & [ 1.25510$]$ & [-1.58289] & {$[-1.71680]$} \\
\hline \multirow[t]{3}{*}{ D(TOT(-2)) } & $6.64 \mathrm{E}-13$ & -0.001961 & $9.78 \mathrm{E}-13$ & $8.12 \mathrm{E}-11$ & $-5.97 \mathrm{E}-12$ & $4.87 \mathrm{E}-12$ & $2.70 \mathrm{E}-11$ & -0.332849 \\
\hline & $(6.8 \mathrm{E}-13)$ & $(0.00618)$ & $(2.4 \mathrm{E}-11)$ & $(5.0 \mathrm{E}-11)$ & $(1.0 \mathrm{E}-11)$ & $(2.5 \mathrm{E}-11)$ & $(2.9 \mathrm{E}-11)$ & $(0.30593)$ \\
\hline & [ 0.98231$]$ & {$[-0.31726]$} & [ 0.03995$]$ & [ 1.63334$]$ & {$[-0.59110]$} & [0.19802] & [ 0.91926$]$ & {$[-1.08801]$} \\
\hline \multirow[t]{3}{*}{$\mathrm{C}$} & 0.021898 & $2.78 \mathrm{E}+08$ & -0.095895 & 0.784066 & 0.042182 & 0.490943 & 0.011709 & $3.56 \mathrm{E}+08$ \\
\hline & $(0.01281)$ & $(1.2 \mathrm{E}+08)$ & $(0.46344)$ & $(0.94130)$ & $(0.19122)$ & $(0.46596)$ & $(0.55587)$ & $(5.8 \mathrm{E}+09)$ \\
\hline & [-1.70937] & [ 2.37589] & {$[-0.20692]$} & [ 0.83296$]$ & [ 0.22060$]$ & [ 1.05362$]$ & [ 0.02106$]$ & [ 0.06140$]$ \\
\hline R-squared & 0.875244 & 0.800135 & 0.598760 & 0.707579 & 0.567208 & 0.475675 & 0.685300 & 0.412919 \\
\hline Adj. R-squared & 0.763620 & 0.621308 & 0.239756 & 0.445939 & 0.179973 & 0.006543 & 0.403726 & -0.112364 \\
\hline Sum sq. resids & 0.054939 & $4.59 \mathrm{E}+18$ & 71.90305 & 296.6308 & 12.24136 & 72.68637 & 103.4460 & $1.12 \mathrm{E}+22$ \\
\hline S.E. equation & 0.053773 & $4.91 \mathrm{E}+08$ & 1.945346 & 3.951221 & 0.802672 & 1.955914 & 2.333351 & $2.43 \mathrm{E}+10$ \\
\hline F-statistic & 7.840998 & 4.474357 & 1.667835 & 2.704401 & 1.464764 & 1.013946 & 2.433817 & 0.786088 \\
\hline Log likelihood & 67.97950 & -780.6404 & -64.79214 & -91.00978 & -32.03793 & -64.99259 & -71.52116 & -925.0131 \\
\hline Akaike AIC & -2.701594 & 43.16975 & 4.475251 & 5.892420 & 2.704753 & 4.486086 & 4.838982 & 50.97368 \\
\hline Schwarz SC & -1.917905 & 43.95344 & 5.258941 & 6.676110 & 3.488443 & 5.269776 & 5.622671 & 51.75737 \\
\hline Mean dependent & -0.037027 & $1.83 \mathrm{E}+08$ & -0.305092 & -0.053979 & -0.001351 & 0.498366 & -0.201380 & $-1.44 \mathrm{E}+09$ \\
\hline S.D. dependent & 0.110601 & $7.98 \mathrm{E}+08$ & 2.231106 & 5.308265 & 0.886388 & 1.962344 & 3.021739 & $2.31 \mathrm{E}+10$ \\
\hline \multicolumn{3}{|c|}{ Determinant resid covariance (dof adj.) } & \multicolumn{2}{|c|}{$1.45 \mathrm{E}+36$} & & & & \\
\hline \multicolumn{3}{|c|}{ Determinant resid covariance } & \multicolumn{2}{|c|}{$7.01 \mathrm{E}+33$} & & & & \\
\hline \multicolumn{3}{|l|}{ Log likelihood } & \multicolumn{2}{|c|}{-1861.762} & & & & \\
\hline \multicolumn{3}{|c|}{ Akaike information criterion } & \multicolumn{2}{|c|}{108.8520} & & & & \\
\hline \multicolumn{3}{|l|}{ Schwarz criterion } & \multicolumn{2}{|c|}{115.4698} & & & & \\
\hline \multicolumn{3}{|l|}{ Number of coefficients } & \multicolumn{2}{|c|}{152} & & & & \\
\hline
\end{tabular}

\subsection{Test the Quality of the Model}

In order to test the quality of the model, the researcher tested the normal distribution of the Residual, which is shown in Table 6, where it was found that the Residual is distributed naturally. The null hypothesis is assumed thatthe Residual follow the natural distribution. The researcher tested the residual serial correlation; the results of thistest in the table 7 indicated that there is no residual serial correlation between the errors in order to accept the null hypothesis that suggests the independence of random errors. The Heteroskedasticity test presented in Table 8 hasbeen accepted for the null hypothesis, which assumes the homoscedasticity of the study variables and rejects the Heteroskedasticity. Also Wald Test results in table 9 illustrate the significance of all study variables. From previous model quality tests, it is clear that the model is acceptable and can be relied on.

Table 6. VEC Residual Normality Tests result

Orthogonalization: Cholesky (Lutkepohl)

Null Hypothesis: Residuals are multivariate normal Sample: 19802019 Included bservations:37

\begin{tabular}{lcccc} 
Component & Skewness & Chi-sq & df & Prob.* $^{*}$ \\
\hline 1 & 0.073165 & 0.033011 & 1 & 0.8558 \\
2 & -0.396474 & 0.969348 & 1 & 0.3248 \\
3 & 0.055639 & 0.019090 & 1 & 0.8901 \\
4 & -0.276932 & 0.472929 & 1 & 0.4916 \\
5 & -0.183628 & 0.207935 & 1 & 0.6484 \\
6 & 0.151318 & 0.141198 & 1 & 0.7071 \\
7 & -0.547155 & 1.846168 & 1 & 0.1742 \\
8 & 0.376072 & 0.872153 & 1 & 0.3504 \\
\hline Joint & & 4.561833 & 8 & 0.8032 \\
\hline
\end{tabular}




\begin{tabular}{lcccc}
\hline Component & Kurtosis & Chi-sq & df & Prob. \\
\hline 1 & 2.859015 & 0.030644 & 1 & 0.8610 \\
2 & 4.230105 & 2.332786 & 1 & 0.1267 \\
3 & 2.389228 & 0.575108 & 1 & 0.4482 \\
4 & 3.152320 & 0.035769 & 1 & 0.8500 \\
5 & 3.116423 & 0.020896 & 1 & 0.8851 \\
6 & 3.250144 & 0.096465 & 1 & 0.7561 \\
7 & 4.021635 & 1.609096 & 1 & 0.2046 \\
8 & 3.697286 & 0.749570 & 1 & 0.3866 \\
\hline Joint & & 5.450333 & 8 & 0.7085 \\
\hline Component & Jarque-Bera & df & Prob. & \\
\hline 1 & 0.063654 & 2 & 0.9687 & 0.1918 \\
2 & 3.302134 & 2 & 0.7430 \\
3 & 0.594198 & 2 & 0.7754 & \\
4 & 0.508698 & 2 & 0.8919 \\
5 & 0.228831 & 2 & 0.8880 \\
6 & 0.237663 & 2 & 0.1777 \\
7 & 3.455264 & 2 & 0.4445 \\
\hline Joint & 1.621723 & 2 & 0.8660 \\
\hline Approximate p-values do not account for coefficient estimation. & &
\end{tabular}

Table 7. VEC residual serial correlation LM tests

\begin{tabular}{|c|c|c|c|c|c|c|}
\hline \multicolumn{7}{|c|}{ Null hypothesis: No serial correlation at lag h } \\
\hline Lag & LRE* stat & df & Prob. & Rao F-stat & df & Prob. \\
\hline 1 & 69.51626 & 64 & 0.2971 & 0.993758 & $(64,29.6)$ & 0.5233 \\
\hline 2 & 77.08978 & 64 & 0.1262 & 1.187665 & $(64,29.6)$ & 0.3088 \\
\hline 3 & 76.77634 & 64 & 0.1314 & 1.179150 & $(64,29.6)$ & 0.3167 \\
\hline \multicolumn{7}{|c|}{ Null hypothesis: No serial correlation at lags 1 to $h$} \\
\hline Lag & LRE* stat & df & Prob. & Rao F-stat & df & Prob. \\
\hline 1 & 69.51626 & 64 & 0.2971 & 0.993758 & $(64,29.6)$ & 0.5233 \\
\hline 2 & 1275.864 & 128 & 0.0000 & NA & $(128, \mathrm{NA})$ & NA \\
\hline 3 & NA & 192 & NA & NA & $(192, \mathrm{NA})$ & NA \\
\hline
\end{tabular}

*Edgeworth expansion corrected likelihood ratio statistic.

Table 8. VEC Residual Heteroskedasticity Tests (Levels and Squares)

\begin{tabular}{|c|c|c|c|c|c|}
\hline \multicolumn{6}{|l|}{ Joint test: } \\
\hline Chi-sq & & df & & Prob. & \\
\hline \multirow[t]{2}{*}{1258.000} & & 1224 & & 0.2437 & \\
\hline & \multicolumn{5}{|c|}{ Individual components: } \\
\hline Dependent & R-squared & $\mathrm{F}(34,2)$ & Prob. & Chi-sq(34) & Prob. \\
\hline res1*res1 & 0.936298 & 0.864597 & 0.6734 & 34.64303 & 0.4371 \\
\hline res $2 *$ res 2 & 0.956389 & 1.289998 & 0.5314 & 35.38639 & 0.4026 \\
\hline res $3 *$ res 3 & 0.961125 & 1.454308 & 0.4904 & 35.56161 & 0.3947 \\
\hline res $4 *$ res 4 & 0.968632 & 1.816451 & 0.4183 & 35.83939 & 0.3822 \\
\hline res $5 *$ res 5 & 0.986674 & 4.355526 & 0.2039 & 36.50696 & 0.3530 \\
\hline res6*res6 & 0.817963 & 0.264316 & 0.9672 & 30.26462 & 0.6514 \\
\hline res7*res7 & 0.753680 & 0.179986 & 0.9918 & 27.88617 & 0.7608 \\
\hline res $8 *$ res8 & 0.903570 & 0.551190 & 0.8216 & 33.43210 & 0.4953 \\
\hline res $2 * \operatorname{res} 1$ & 0.897255 & 0.513694 & 0.8417 & 33.19842 & 0.5067 \\
\hline res3*res1 & 0.968853 & 1.829744 & 0.4160 & 35.84755 & 0.3818 \\
\hline res $3 *$ res 2 & 0.851582 & 0.337513 & 0.9349 & 31.50853 & 0.5903 \\
\hline res4*res1 & 0.960900 & 1.445613 & 0.4924 & 35.55330 & 0.3950 \\
\hline res4*res2 & 0.937492 & 0.882235 & 0.6662 & 34.68721 & 0.4350 \\
\hline res4*res3 & 0.982128 & 3.232635 & 0.2640 & 36.33875 & 0.3602 \\
\hline res5*res1 & 0.972238 & 2.060004 & 0.3804 & 35.97279 & 0.3763 \\
\hline res5*res 2 & 0.995053 & 11.83287 & 0.0808 & 36.81698 & 0.3398 \\
\hline
\end{tabular}




\begin{tabular}{llllll}
\hline res5*res3 & 0.990290 & 5.999246 & 0.1528 & 36.64073 & 0.3473 \\
res5*res4 & 0.967874 & 1.772224 & 0.4260 & 35.81135 & 0.3834 \\
res6*res1 & 0.904245 & 0.555492 & 0.8193 & 33.45708 & 0.4941 \\
res6*res2 & 0.902724 & 0.545882 & 0.8244 & 33.40078 & 0.4968 \\
res6*res3 & 0.942059 & 0.956406 & 0.6375 & 34.85618 & 0.4271 \\
res6*res4 & 0.963488 & 1.552246 & 0.4686 & 35.64905 & 0.3907 \\
res6*res5 & 0.953109 & 1.195639 & 0.5580 & 35.26502 & 0.4082 \\
res7*res1 & 0.863138 & 0.370978 & 0.9181 & 31.93611 & 0.5691 \\
res7*res2 & 0.875178 & 0.412437 & 0.8963 & 32.38160 & 0.5470 \\
res7*res3 & 0.934926 & 0.845126 & 0.6814 & 34.59227 & 0.4395 \\
res7*res4 & 0.984690 & 3.783431 & 0.2307 & 36.43354 & 0.3561 \\
res7*res5 & 0.934809 & 0.843504 & 0.6821 & 34.58794 & 0.4397 \\
res7*res6 & 0.871540 & 0.399089 & 0.9034 & 32.24697 & 0.5537 \\
res8*res1 & 0.874962 & 0.411620 & 0.8968 & 32.37358 & 0.5474 \\
res8*res2 & 0.885348 & 0.454239 & 0.8738 & 32.75788 & 0.5284 \\
res8*res3 & 0.785959 & 0.706202 & 0.9833 & 29.08049 & 0.7075 \\
res8*res4 & 0.923109 & 6.816583 & 0.7434 & 34.15504 & 0.4603 \\
res8*res5 & 0.991444 & 0.263511 & 0.1359 & 36.68344 & 0.3454 \\
res8*res6 & 0.817508 & 0.198313 & 0.9675 & 30.24780 & 0.6522 \\
res8*res7 & 0.771237 & & 0.9879 & 28.53575 & 0.7323 \\
\hline
\end{tabular}

Table 9. Wald Test result

\begin{tabular}{lccc}
\hline \multicolumn{2}{l}{ Wald Test: Equation: Untitled } & \multicolumn{3}{c}{ Value } & df & Probability \\
\hline Test Statistic & 63.27229 & $(8,29)$ & 0.0000 \\
\hline F-statistic & 506.1783 & 8 & 0.0000 \\
Chi-square & & \\
\hline Null Hypothesis: $C(1)=0, C(2)=C(3)=C(4)=C(5)=C(6)=C(7)=C(8)=0$ & & \\
\hline
\end{tabular}

\begin{tabular}{lcc}
\hline Null Hypothesis Summary: & & \\
\hline Normalized Restriction $(=0)$ & Value & Std. Err. \\
\hline $\mathrm{C}(1)$ & $-2.92 \mathrm{E}-11$ & $5.76 \mathrm{E}-12$ \\
$\mathrm{C}(2)$ & -0.010591 & 0.001770 \\
$\mathrm{C}(3)$ & 0.002546 & 0.000706 \\
$\mathrm{C}(4)$ & 0.036847 & 0.003841 \\
$\mathrm{C}(5)$ & -0.050204 & 0.002701 \\
$\mathrm{C}(6)$ & 0.016536 & 0.001533 \\
$\mathrm{C}(7)$ & $-1.96 \mathrm{E}-12$ & $1.99 \mathrm{E}-13$ \\
$\mathrm{C}(8)$ & $1.04 \mathrm{E}-17$ & 0.001666 \\
\hline
\end{tabular}

Restrictions are linear in coefficients.

\section{Conclusion}

This study examines the estimation of the long-run relationship between the external debt service and the exchange rate in Egypt in the period 1980-2019 and relies on the exchange rate of the dollar against the Egyptian pound as a dependent variable, while the explanatory variables were the external debt service, gross capital formation, broad money growth, deposit interest rate, household final consumption expenditure, gross savings, and terms of trade adjustment. This study methodology is based on Vector Error Correction (VEC) and concludes that there is a significant long-term relationship between the value of the Egyptian pound and all the variables explained in the study, as the error correction coefficient is negative and significant. Also, there is an inverse statistically significant relationship between the value of the Egyptian pound and each of the external debt service, the deposit interest rate, and gross savings; any change of $1 \%$ in the external debt service, the deposit interest rate, and gross savings leads to a devaluation of the Egyptian pound against the dollar by $4.8 \%$, $0.04 \%$, and $0.05 \%$, respectively. The study also concluded that there is a positive, statistically significant relationship in the long term between the value of the Egyptian pound and each of gross capital formation, broad money growth, households' and NPISHs' final consumption expenditure, and terms of trade adjustment, as any change of $1 \%$ in these variables leads to an increase in the value of the Egyptian pound by $0.16 \%, 0.05 \%, 0.27 \%$, and $6 \%$, respectively. This study recommends that decision makers consider all the reasons that would reduce the external debt service in order to preserve the value of the Egyptian currency in the long run. 


\section{References}

Aderemi, T. A., Fagbola, L. O., Sokunbi, G. M., \& Ebere, C. E. (2020). Investigating External Debt and Exchange Rate Fluctuations in Nigeria: Any Difference with ARDL Model? Studia Universitatis Babes-Bolyai Oeconomica, 65(3), 53-64. https://doi.org/10.2478/subboec-2020-0015

Ajayi, R. A., \& Jongmoo, J. C. (1993) .The effect of foreign debt on currency values. Journal of Economics and Business, 45(3-4), 331-340. https://doi.org/10.1016/0148-6195(93)90021-F

Alam, N., \& Fauziah, M. T. (2013). An Investigation of The Relationship of External Public Debt with Budget Deficit, Current Account Deficit, and Exchange Rate Depreciation in Debt Trap and Non-Debt Trap Countries. European Scientific Journal, 9(22), 144-158. https://doi.org/10.19044/esj.2013.v9n22p\%25p

Beaugrand, P., Bolieau, L., \& Montfort, M. (2002). The Choice Between External and Domestic Debt in Financing Budget Deficits: The Case of Central and West African Countries. IMF Working Paper, 02(79). https://doi.org/10.5089/9781451850215.001

Bua, G., Pradelli, J., \& Presbitero, A. (2014). Domestic public debt in Low-Income Countries: Trends and structure. Review of Development Finance, 4(1), 1-19. https://doi.org/10.1016/j.rdf.2014.02.002

Cavallo, E., Powell, A., Pedemonte, M., \& Tavella, P. (2013). A New Taxonomy of Sudden Stops: Which Sudden Stops Should Countries Be Most Concerned About? Inter-American Development Bank, working paper, August. https://doi.org/10.2139/ssrn.2367696

Claude, K., \& Dany, D. (2019). External Debts and Real Exchange Rates in Developing Countries: Evidence from Chad. Sumerianz Journal of Economics and Finance, 2(1), 1-6. Retrieved from https://www.sumerianz.com/pdf-files/sjef2(1)1-6.pdf

Eichengreen, B., Hausmann, R., \& Panizza, U. (2005). The Pain of Original Sin. Chicago University Press. https://doi.org/10.7208/chicago/9780226194578.003.0002

Mutua, D. K., \& Mugendi, C. N. (2020). The External Debt Service and Exchange Rate in Kenya. International Journal of Scientific Research and Innovative Technology, 7(10), 37-56. Retrieved from https://www.ijsrit.com/uploaded_all_files/2496763157_x3.pdf

Quilent, A. O. (2015). An Analysis on the Effect of External Public Debt on Exchange Rate Volatility in Kenya. Thesis, University of Nairobi. Retrieved from http://erepository.uonbi.ac.ke/handle/11295/95259

Titus, F. I. N., \& Richard, E. (2015). Assessing the Effect of External Debt Servicing and Receipt on Exchange Rate in Nigeria. International Journal of Economics and Finance, 7(9), 278-286. https://doi.org/10.5539/ijef.v7n9p278

\section{Copyrights}

Copyright for this article is retained by the author(s), with first publication rights granted to the journal.

This is an open-access article distributed under the terms and conditions of the Creative Commons Attribution license (http://creativecommons.org/licenses/by/4.0/). 